\title{
A case of generalized lipodystrophy-associated progeroid syndrome treated by leptin replacement with short and long-term monitoring of the metabolic and endocrine profiles
}

\author{
Takahiro Fukaishi ${ }^{1)}$, Isao Minami ${ }^{1)}$, Seizaburo Masuda ${ }^{1)}$, Yasutaka Miyachi ${ }^{1)}$, Kazutaka Tsujimoto ${ }^{1)}$, \\ Hajime Izumiyama ${ }^{1), 2)}$, Koshi Hashimoto ${ }^{3), 4)}$, Masayuki Yoshida ${ }^{5)}$, Sayako Takahashi ${ }^{5)}$, \\ Kenichi Kashimada ${ }^{6}$, Tomohiro Morio ${ }^{6}$, Kenjiro Kosaki ${ }^{7)}$, Yoshiro Maezawa ${ }^{8)}$, Koutaro Yokote ${ }^{8)}$, \\ Takanobu Yoshimoto ${ }^{1)}$ and Tetsuya Yamada ${ }^{1)}$
}

\footnotetext{
1) Department of Molecular Endocrinology and Metabolism, Tokyo Medical and Dental University, Bunkyo-ku, Tokyo 113-8510, Japan

2) Center for Medical Welfare and Liaison Services, Tokyo Medical and Dental University, Bunkyo-ku, Tokyo 113-8510, Japan

3) Department of Preemptive Medicine and Metabolism, Graduate School of Medical and Dental Sciences, Tokyo Medical and Dental University, Bunkyo-ku, Tokyo 113-8510, Japan

4) Department of Diabetes, Endocrinology and Hematology, Dokkyo Medical University Saitama Medical Center, Koshigaya, Saitama 343-8555, Japan

5) Division of Medical Genetics, Medical Hospital of Tokyo Medical and Dental University, Bunkyo-ku, Tokyo 113-8510, Japan

6) Department of Pediatrics and Developmental Biology, Tokyo Medical and Dental University, Bunkyo-ku, Tokyo 113-8510, Japan

7) Center for Medical Genetics, Keio University School of Medicine, Shinjuku-ku, Tokyo 160-0016, Japan

${ }^{8)}$ Department of Endocrinology, Hematology and Gerontology, Graduate School of Medicine, Chiba University, Chiba, Chiba 260-0856, Japan
}

\begin{abstract}
We herein report a case of a 28-year-old man with generalized lipodystrophy-associated progeroid syndrome treated by leptin replacement. He showed symptoms of generalized lipodystrophy around onset of puberty. His body mass index was $11.9 \mathrm{~kg} / \mathrm{m}^{2}$, and he had a short stature, birdlike facies, dental crowding due to micrognathia, partial graying and loss of hair, and a high-pitched voice, all of which are typical features of the progeroid syndrome. Laboratory examinations and abdominal ultrasonography revealed diabetes mellitus, insulin-resistance, dyslipidemia, decreased serum leptin levels ( $2.2 \mathrm{ng} /$ $\mathrm{mL}$ ), elevated serum hepatobiliary enzyme levels and fatty liver. Whole exome sequencing revealed de novo heterozygous LMNA p.T10I mutation, indicating generalized lipodystrophy-associated progeroid syndrome, which is a newly identified subtype of atypical progeroid syndrome characterized by severe metabolic abnormalities. Daily injection of metreleptin [1.2 $\mathrm{mg}(0.04 \mathrm{mg} / \mathrm{kg}) / \mathrm{day}$ ] was started. Metreleptin treatment significantly improved his diabetes from $\mathrm{HbA} 1 \mathrm{c} 11.0 \%$ to $5.4 \%$ in six months. It also elevated serum testosterone levels. Elevated serum testosterone levels persisted even 1 year after the initiation of metreleptin treatment. To the best of our knowledge, this is the first Japanese case report of generalized lipodystrophy-associated progeroid syndrome. Furthermore, we evaluated short and long-term effectiveness of leptin replacement on generalized lipodystrophy by monitoring metabolic and endocrine profiles.
\end{abstract}

Key words: Generalized lipodystrophy-associated progeroid syndrome, LMNA gene, Leptin, Testosterone

LIPODYSTROPHY is a rare disease, its prevalence being estimated to be approximately $1.3-4.7$ cases/

Submitted Jun. 5, 2019; Accepted Oct. 15, 2019 as EJ19-0226 Released online in J-STAGE as advance publication Nov. 8, 2019 Correspondence to: Takahiro Fukaishi, M.D., Department of Molecular Endocrinology and Metabolism, Tokyo Medical and Dental University, 1-5-45 Yushima, Bunkyo-ku, Tokyo, 113-8510, Japan.

E-mail: fukaishi.mem@tmd.ac.jp million [1]. These patients show either near complete or partial lack of adipose tissue. There are four types of lipodystrophy; congenital generalized lipodystrophy (CGL), familial partial lipodystrophy (FPLD), acquired generalized lipodystrophy (AGL) and acquired partial lipodystrophy (APL) [2]. The inheritance of CGL is autosomal recessive while FPLD is autosomal dominant, and AGL and APL are more common among females. Patients with generalized lipodystrophy have leptin defi- 
ciency secondary to a near total loss of adipose tissue, which is associated with a severe form of metabolic disorders characterized by dyslipidemia, fatty liver, insulin resistance and diabetes.

Mutations in the lamin A/C (LMNA) gene cause progeroid syndromes, which often accompany generalized lipodystrophy. They are known as autosomal dominant Hutchinson-Gilford progeria syndrome (HGPS) [3], atypical progeroid syndrome (APS) including atypical Werner syndrome [4] and autosomal recessive mandibuloacral dysplasia [5]. The prevalence is extremely low, and there is no apparent sex difference.

Recently, Hussain et al. reported some APS cases with de novo heterozygous $L M N A$ c.29C $>$ T; p.T10I mutation. These patients had clinical features distinct from other types of APS that include severe metabolic disorders such as diabetes mellitus, dyslipidemia and nonalcoholic fatty liver disease. They therefore named this novel syndrome as a generalized lipodystrophy-associated progeroid syndrome (GLPS) [6].

Metreleptin replacement therapy dramatically improves insulin resistance and dyslipidemia in patients with generalized lipodystrophy [7, 8]. However, the effects of metreleptin on testosterone as well as its effect on skeletal muscle mass remain unclear. We herein report a case of GLPS with metabolic abnormalities and describe the changes in appetite, hormones and body composition right after (shortly after, two weeks after, etc) and also one year after the initiation of metreleptin treatment.

\section{Case Report}

A 28-year-old Japanese man was admitted to our hospital because of lipodystrophy. He had normal height $(51.5 \mathrm{~cm})$ and weight $(3,296 \mathrm{~g})$ at birth. Moderate physical growth retardation was seen as early as one month of age, but no apparent mental or hormonal disorders were seen at that point in time (Figs. 1, 2). The generalized lipodystrophy became overt around onset of puberty, but a medical examination when he was around 20 years of age showed no metabolic abnormalities. From 25 years of age, he developed increased thirst, polydipsia and polyuria and visited the general internal medicine clinic at the age of 28. Subsequent examinations revealed that he had already developed hyperglycemia and dyslipidemia (HbA1c: 9.7-10.1\%, HOMA-IR: 5.64, TG: 281-783 mg/ dL). Up till that point, he had no significant past medical history, no known family history, and no consanguinity in his relatives (Fig. 3). His height, body weight and body mass index were $153.6 \mathrm{~cm}, 28.3 \mathrm{~kg}$ and $11.9 \mathrm{~kg} / \mathrm{m}^{2}$, respectively. He had a short stature, birdlike facies with a beaked nose, dental crowding due to micrognathia, partial graying and loss of hair, and a high-pitched voice, all of which are typical features of the APS. There were no significant skin abnormalities and joint contractures. Table 1 shows the laboratory data on admission (fasting). His glycated hemoglobin (HbA1c) was $11.0 \%$. Serum aspartate transaminase (AST), alanine aminotransferase (ALT) and $\gamma$-glutamyltransferase $(\gamma$-GTP) levels were 40 , 81 and $65 \mathrm{IU} / \mathrm{L}$, respectively, indicating moderate liver

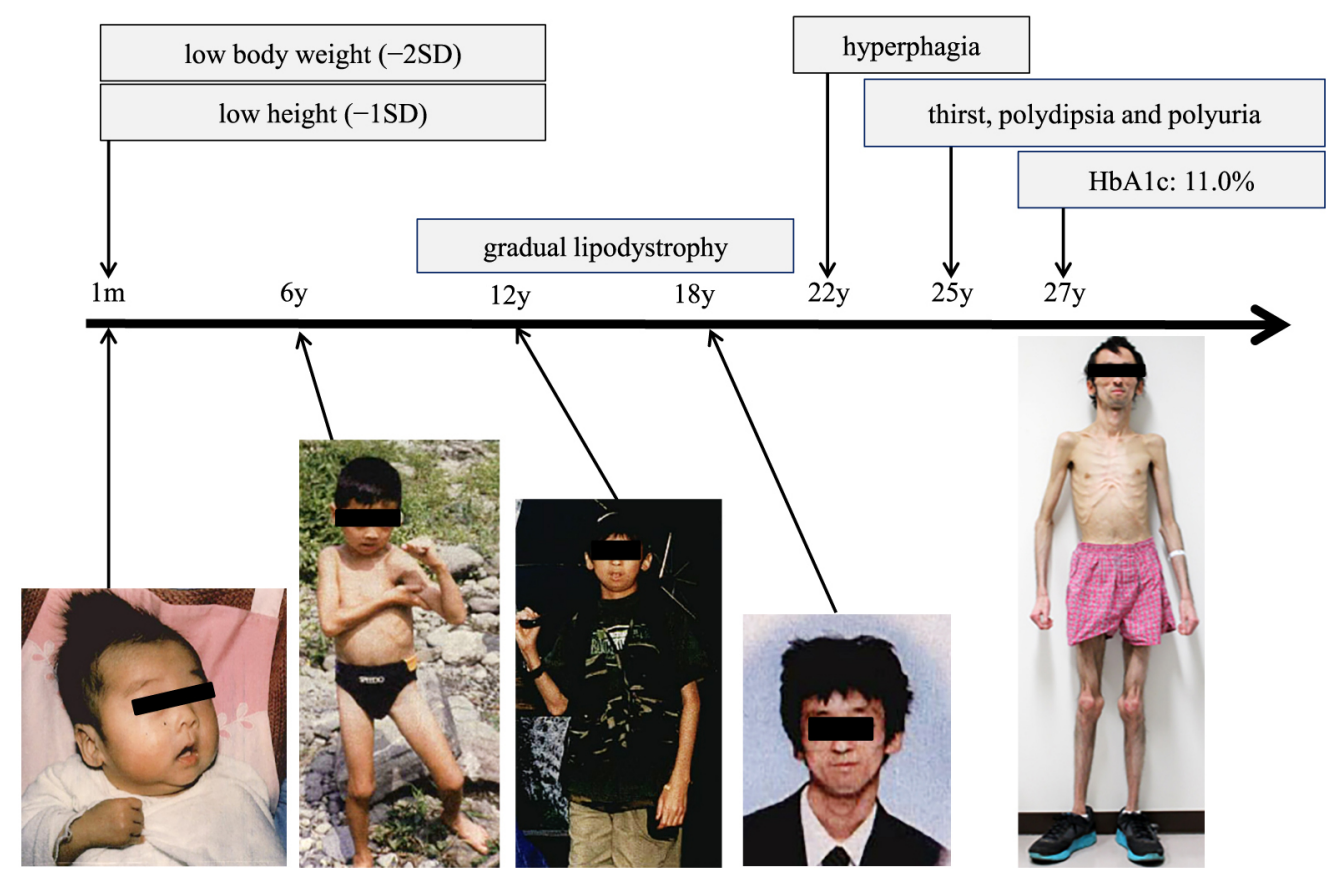

Fig. 1 Time course of growth and clinical findings of the present case. 


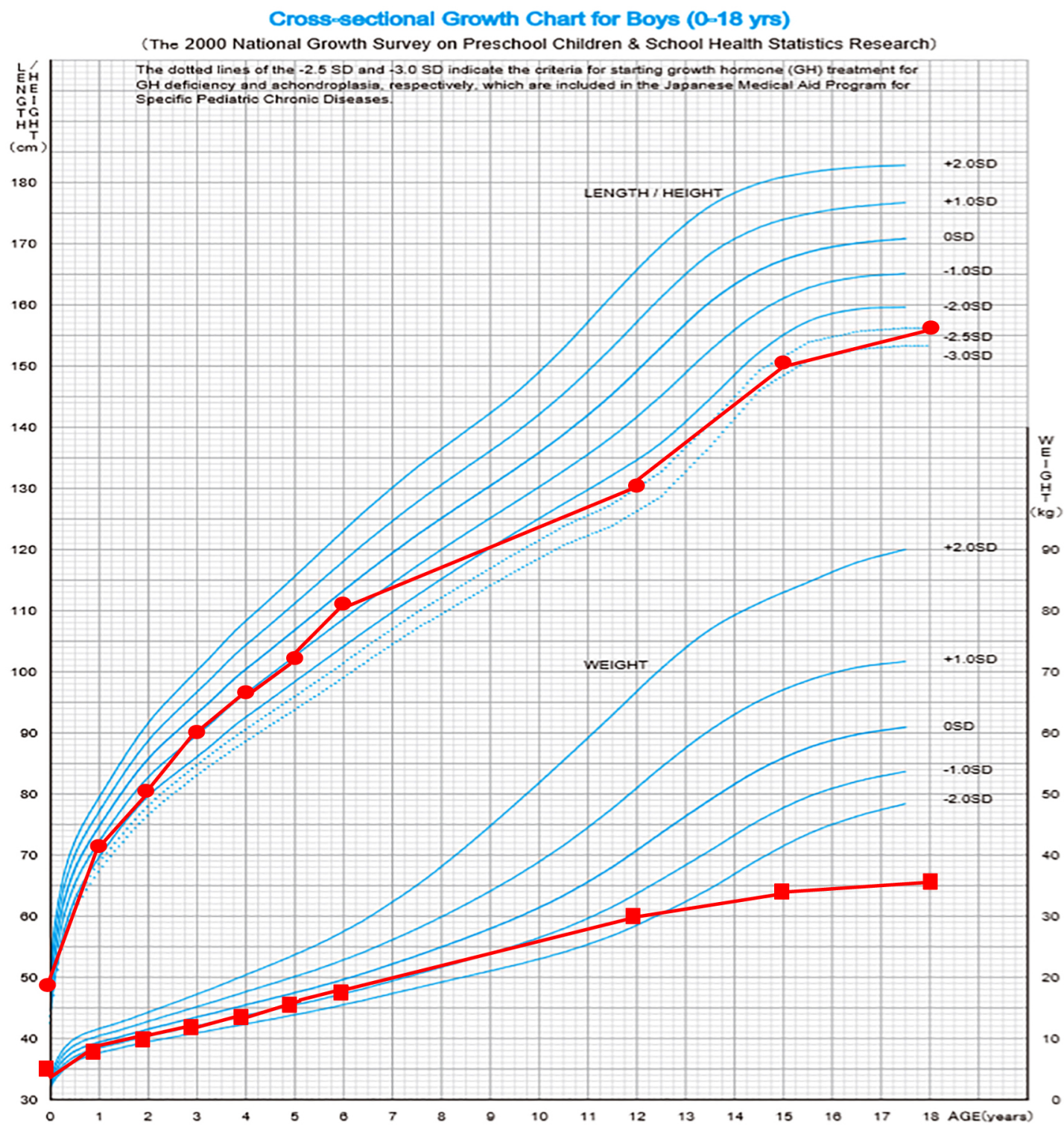

Fig. 2 Patient's growth chart from birth to 18 years of age. Closed red circles indicate height (top panel) and closed red squares indicate body weight (bottom panel).

dysfunction. Serum triglyceride (TG) levels were 158 $\mathrm{mg} / \mathrm{dL}$. He had decreased serum leptin levels $(2.2$ $\mathrm{ng} / \mathrm{mL}$, normal: $2.4-25.0)$ and decreased serum total testosterone levels $(1.90 \mathrm{ng} / \mathrm{mL})$. His secondary sexual characteristics were normal. Lack of pubic or axillary hair, decreased libido and dysfunction of erection or ejaculation were not present. Abdominal ultrasonography showed a fatty liver, and magnetic resonance imaging showed the absence of both subcutaneous and visceral body fat (Fig. 4). Chest X-ray showed scoliosis. Whole exome sequencing revealed a de novo heterozygous LMNA p.T10I mutation (Fig. 5), indicating that he had GLPS. Whole exome sequencing of his parents was also performed and no mutation was detected. Electrocardiogram and ultracardiography were performed because patients with GLPS often suffer from cardiovascular diseases, but neither examination revealed any cardiac abnormality. Appropriate diet therapy after hospitalization immediately improved his blood glucose significantly, but it seemed difficult to continue diet therapy outside the hospital due to hyperphagia. Daily injection of metreleptin $[1.2 \mathrm{mg}(0.04 \mathrm{mg} / \mathrm{kg}) / \mathrm{day}]$ was started. Shortly after metreleptin replacement, he felt decreased hunger sensations before meals and improved satiety sensation after. On the $13^{\text {th }}$ day of treatment, significant improvement in diurnal variation of serum glucose, triglyceride, insulin and plasma glucagon levels were seen (Fig. 6). Furthermore, elevated serum testosterone levels were seen $(4.15 \mathrm{ng} / \mathrm{mL})$. On the other hand, serum luteinizing hormone (LH) levels were slightly increased, 


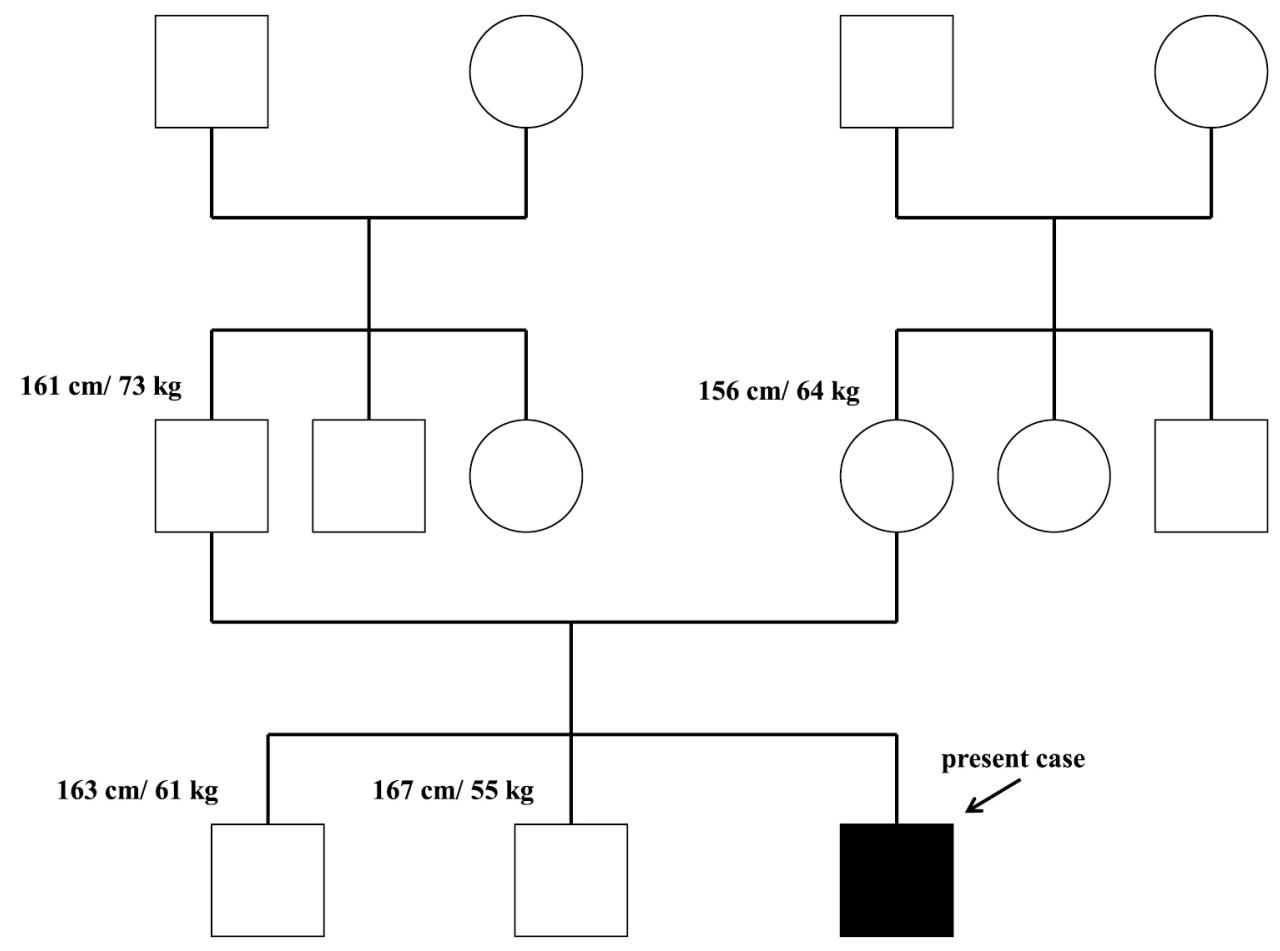

Fig. 3 Family pedigree of the present case.

Table 1 Laboratory data on admission (fasting)

\begin{tabular}{|c|c|c|c|c|c|}
\hline \multicolumn{2}{|l|}{ Complete blood count } & \multicolumn{2}{|l|}{ Urianalysis } & \multicolumn{2}{|c|}{ Hormonal analysis } \\
\hline White blood cells & $10,100 / \mu \mathrm{L}$ & Protein & $(1+)$ & $\mathrm{GH}$ & $4.68 \mathrm{ng} / \mathrm{mL}$ \\
\hline Red blood cells & $485 \times 10^{4} / \mu \mathrm{L}$ & Glucose & $(-)$ & IGF-1 & $189 \mathrm{ng} / \mathrm{mL}$ \\
\hline Hemoglobin & $13.7 \mathrm{~g} / \mathrm{dL}$ & Ketone & $(-)$ & LH & $1.5 \mathrm{mIU} / \mathrm{mL}$ \\
\hline Hematocrit & $39.1 \%$ & CPIR & $141 \mu \mathrm{g} /$ day & FSH & $5.7 \mathrm{mIU} / \mathrm{mL}$ \\
\hline \multirow[t]{2}{*}{ Platelet } & $17.2 \times 10^{4} / \mu \mathrm{L}$ & & & Testosterone & $1.9 \mathrm{ng} / \mathrm{mL}$ \\
\hline & & & & ACTH & $17.4 \mathrm{pg} / \mathrm{mL}$ \\
\hline Blood chemistry & & & & Cortisol & $7.3 \mu \mathrm{g} / \mathrm{dL}$ \\
\hline Albumin & $4.1 \mathrm{~g} / \mathrm{dL}$ & T-chol & $138 \mathrm{mg} / \mathrm{dL}$ & PRL & $13.5 \mathrm{ng} / \mathrm{mL}$ \\
\hline BUN & $7 \mathrm{mg} / \mathrm{dL}$ & TG & $158 \mathrm{mg} / \mathrm{dL}$ & TSH & $0.75 \mu \mathrm{IU} / \mathrm{mL}$ \\
\hline Cre & $0.22 \mathrm{mg} / \mathrm{dL}$ & HDL-chol & $22 \mathrm{mg} / \mathrm{dL}$ & FT4 & $1.09 \mathrm{ng} / \mathrm{dL}$ \\
\hline $\mathrm{Na}$ & $143 \mathrm{mEq} / \mathrm{L}$ & LDL-chol & $87 \mathrm{mg} / \mathrm{dL}$ & Leptin & $2.2 \mathrm{ng} / \mathrm{mL}$ \\
\hline K & $3.4 \mathrm{mEq} / \mathrm{L}$ & Glu & $108 \mathrm{mg} / \mathrm{dL}$ & Adiponectin & $<0.5 \mu \mathrm{g} / \mathrm{mL}$ \\
\hline $\mathrm{Cl}$ & $106 \mathrm{mEq} / \mathrm{L}$ & $\mathrm{HbA} 1 \mathrm{c}$ & $11.0 \%$ & CPIR & $1.6 \mathrm{ng} / \mathrm{mL}$ \\
\hline $\mathrm{Ca}$ & $9.5 \mathrm{mg} / \mathrm{dL}$ & GAD-Ab & $(-)$ & IRI & $8.4 \mu \mathrm{IU} / \mathrm{mL}$ \\
\hline AST & $40 \mathrm{IU} / \mathrm{L}$ & TPO-Ab & $(-)$ & IRG & $111 \mathrm{pg} / \mathrm{mL}$ \\
\hline ALT & $81 \mathrm{IU} / \mathrm{L}$ & $\mathrm{Tg}-\mathrm{Ab}$ & $(-)$ & & \\
\hline$\gamma$-GTP & $65 \mathrm{IU} / \mathrm{L}$ & HIV-Ab & $(-)$ & & \\
\hline
\end{tabular}

BUN, blood urea nitrogen; Cre, creatinine; AST, aspartate aminotransferase; ALT, alanine aminotransferase; $\gamma$-GTP, $\gamma$-glutamyltransferase; CPIR, C-peptide immunoreactivity; T-chol, total cholesterol; TG, triglyceride; HDL-chol, high-density lipoprotein cholesterol; LDL-chol, low-density lipoprotein cholesterol; Glu, glucose; HbA1c, hemoglobin A1c; GAD-Ab, anti-glutamic acid decarboxylase antibody; TPO$\mathrm{Ab}$, anti-thyroid gland peroxisome antibody; $\mathrm{Tg}-\mathrm{Ab}$, anti-thyroglobulin antibody; HIV-Ab, anti-human immunodeficiency virus antibody; IRI, immunoreactive insulin; IRG, immunoreactive glucagon 


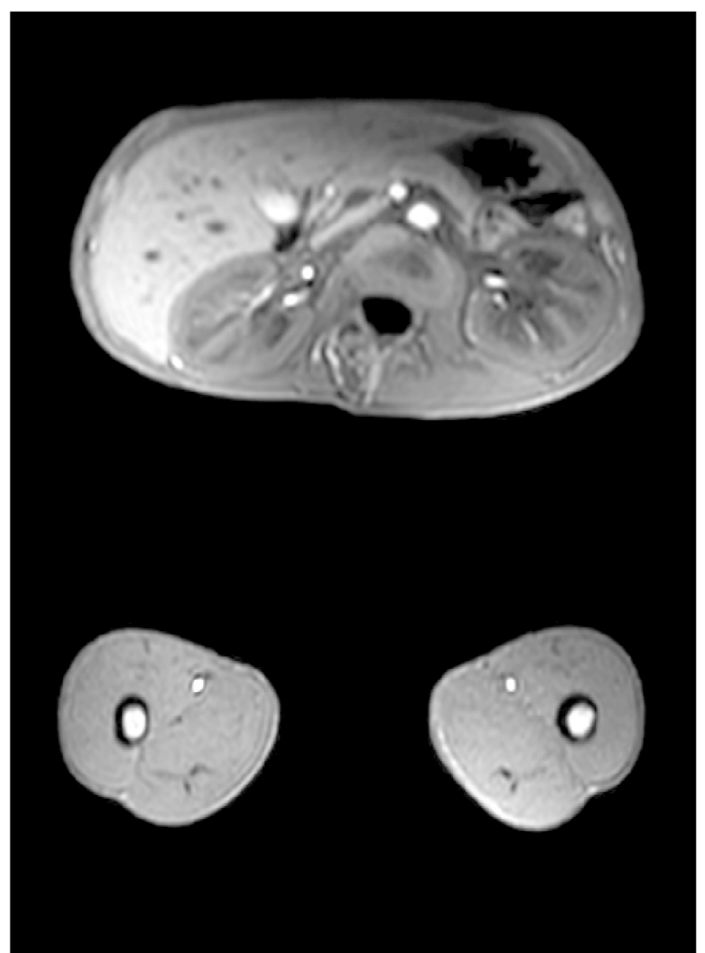

Fig. 4 T-1 weighted magnetic resonance imaging indicating the absence of both subcutaneous and visceral fat.

from $1.5 \mathrm{mIU} / \mathrm{mL}$ to $1.6 \mathrm{mIU} / \mathrm{mL}$. After 1 year of metreleptin injection monotherapy, his HbAlc was 5.3\%, serum TG levels were $86 \mathrm{mg} / \mathrm{dL}$, serum AST levels were $20 \mathrm{IU} / \mathrm{L}$ and serum ALT levels were $12 \mathrm{IU} / \mathrm{L}$, which indicated that his metabolic profiles had been improved by leptin monotherapy. Serum testosterone level (3.73 $\mathrm{ng} / \mathrm{mL}$ ) remained elevated, and LH levels increased (2.7 $\mathrm{mIU} / \mathrm{mL}$ ) after 1 year of metreleptin treatment. Furthermore, a slight increase in skeletal muscle index, from 3.42 to $3.45 \mathrm{~kg} / \mathrm{m}^{2}$, was seen according to dual-energy X-ray absorptiometry (skeletal muscle index; ASM/ height $^{2}$, normal: $>6.87$. ASM; appendicular skeletal muscle mass, the sum of muscle masses of the four limbs).

\section{Discussion}

Generalized lipodystrophy is associated with various kinds of metabolic abnormalities such as diabetes mellitus, dyslipidemia and non-alcoholic fatty liver disease. These metabolic disorders occur as a result of impaired leptin secretion, which results from a near complete lack of adipose tissue [1]. First-line treatment for such metabolic disorders is sufficient diet and exercise therapy, and should this fail to reach efficacy, metreleptin replacement should be considered [2]. The present case demonstrated various metabolic disorders in addition to

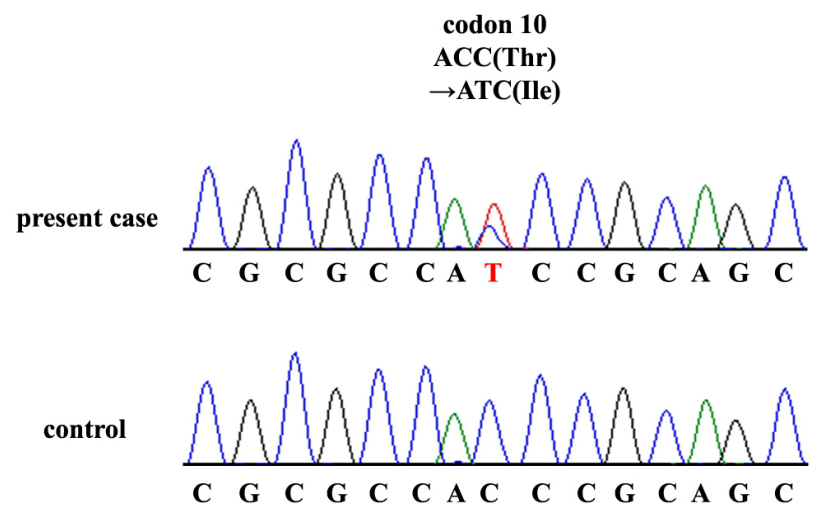

Fig. 5 Exome analyses of the patient and his parents were performed on HiSeq 2500 platform (Illu-mina, CA) and SureSelectXT Human All Exon V6 (Agilent Technologies, Santa Clara, CA). The sequencing reads were aligned to the reference human genome sequence (GRCh37) using Burrows Wheeler Transform. Duplicate reads were then removed using Picard. Local realignment around the indels, base quality score recalibration, and variant calling were performed using the Genome Analysis Toolkit. Non-synonymous coding variants, nonsense coding variants, splice acceptor, donor and predicted site variants and inframe and frameshift coding indels were filtered against dbSNP137, the 1,000 Genomes Project, ESP6500, ExAC, the Integrative Japanese Genome Variation Database and the Human Genetic Variation Browser. The exome data from the patient and his parents were filtered for candidate mutations using DeNovo Check. In this algorithm, variants that were not identified in either parent with more than $3 \%$ variation reads were considered to be candidate de novo mutations. After this filtering process with default parameters, a de novo frameshift mutation in exon 1 of $L M N A$, that is, c.29C $>\mathrm{T}$; p.T10I remained as the only variant in the coding region.

physical features of APS. Therefore, we examined whether he had any gene mutation that could explain both his lipodystrophy and associated metabolic disorders. Whole exome sequencing revealed a de novo heterozygous mutation of $L M N A$ p.T10I, which indicated that he had GLPS, a recently proposed novel subtype of APS [6]. On the other hand, the LMNA mutation also causes other types of lipodystrophy, which should be distinguished from GLPS. Werner syndrome is one of the major progeroid syndromes with metabolic abnormalities [4], but he did not show cataracts or calcification in the Achilles tendon. HGPS is a progeroid syndrome with lipodystrophy caused by $L M N A$ mutation [3], but he did not show baldness, hydrocephalus-like feature and progeria from infancy. Mandibuloacral dysplasia-associated lipodystrophy is known as another type of lipodystrophy caused by LMNA mutation [5], but he did not show clavicle dysplasia, osteolysis in the distal phalanx and joint contractures. Whole exome sequencing did not 
A

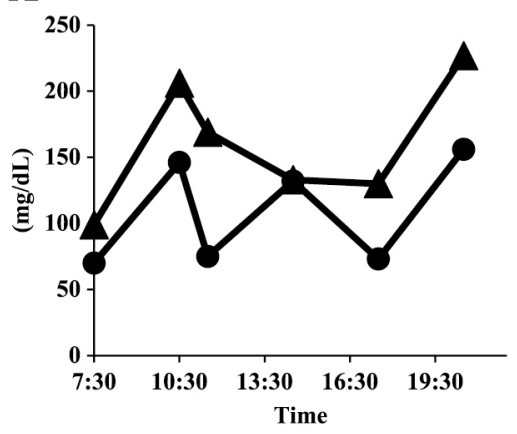

C

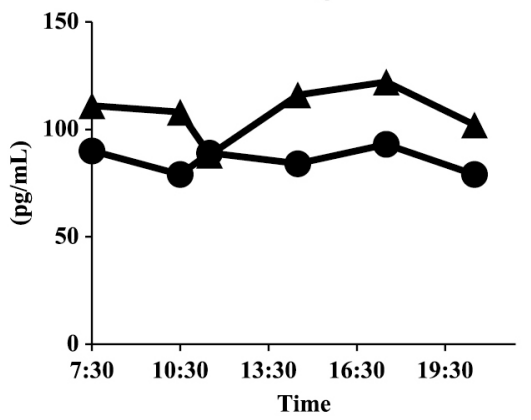

B

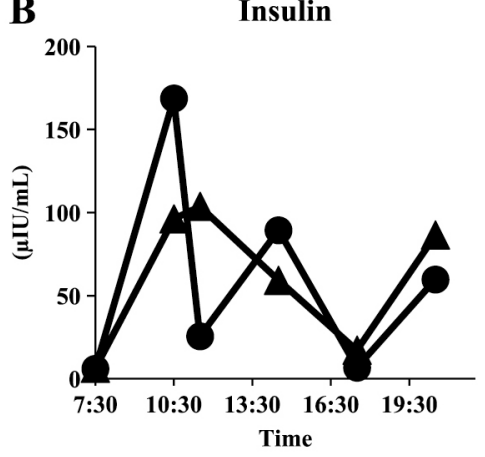

D

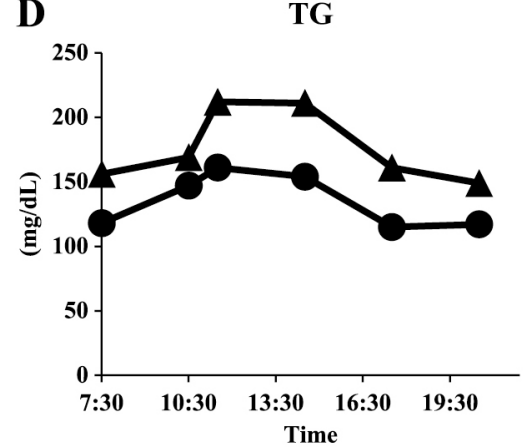

$\mathbf{E}$

\begin{tabular}{|l|r|r|}
\hline $\begin{array}{c}\text { AUC } \\
(\text { Area under the curve) }\end{array}$ & \multicolumn{1}{c|}{ before } & \multicolumn{1}{c|}{ day12 } \\
\hline Glu $(\mathrm{mg} \cdot \mathrm{min} / \mathrm{dL})$ & 121,680 & 83,760 \\
\hline IRI $(\mathrm{uIU} \cdot \mathrm{min} / \mathrm{mL})$ & 46,110 & 46,524 \\
\hline IRG $(\mathrm{pg} \cdot \mathrm{min} / \mathrm{mL})$ & 85,330 & 67,230 \\
\hline TG $(\mathrm{mg} \cdot \mathrm{min} / \mathrm{dL})$ & 140,130 & 106,530 \\
\hline
\end{tabular}

1 before treatment

treatment day12

Fig. 6 Diurnal variations of (A) glucose, (B) insulin, (C) glucagon and (D) triglyceride levels immediately before and 12 days after the initiation of metreleptin treatment. E: Area under the curve of glucose, insulin, glucagon and triglyceride levels.

reveal mutations related to these differential diagnoses.

Eleven patients with GLPS has been reported thus far [6]. Ten out of eleven had a de novo heterozygous mutation of LNMA c.29C>T; p.T10I, the type of mutation seen in the present case. The one case inherited this type of mutation from her father. In most GLPS patients, onset of generalized lipodystrophy occurs in their childhood, and most of them are diagnosed with GLPS between 10 and 20 years of age. Importantly, metabolic parameters are worse in these patients compared to other types of progeroid syndromes and a major cause of morbidity is cardiopathy including coronary artery disease. Considering the high incidence of severe and even fatal metabolic abnormalities and cardiopathy among patients with GLPS, early diagnosis and treatment are important.

Although the present case had presented with physical growth retardation since infancy, symptoms of generalized lipodystrophy appeared around onset of puberty and diabetes seemed to have developed in his adulthood, which indicated that progression of the disease in the present case was relatively slow compared to previous cases. The reason for this difference is unclear, but ethnic difference may have affected the clinical course. However, only limited cases of GLPS have been reported from Asia including Japan.

GLPS causes severe insulin resistance due to leptin deficiency secondary to a near total loss of adipose tis- sue. Administration of metreleptin reportedly improved diabetes and other metabolic abnormalities in most GLPS patients [6]. In the present case, we monitored short-term effects of metreleptin on metabolic parameters such as serum glucose, TG and insulin levels (Fig. 6). The short-term metabolic effects were reportedly reversed by treatment suspension, indicating that long term administration of metreleptin was necessary to maintain improved metabolic phenotypes [9]. Consistent with our study, previous reports showed that long-term metreleptin injection markedly improved diabetes mellitus, hypertriglycemia and liver steatosis [7-9]. Given the decreased postprandial serum glucose levels and increased postprandial insulin levels after metreleptin treatment (Fig. 6), the improvement in insulin secretion might play an important role in these favorable effects. Previous reports showed that pancreatic fat deposition was increased in FPLD and it was inversely related to $\beta$ cell function [10]. In the present case, the fatty liver was improved by metreleptin treatment. It might also have ameliorated ectopic fat in the pancreas caused by leptin deficiency, leading to an improvement in insulin secretion. In addition, plasma glucagon levels were decreased by metreleptin treatment, which might also contribute to improved glycemic control in the present case. Additionally, plasma glucagon levels have been reported to be suppressed in leptin-overexpressing and leptin-treated 
mice $[11,12]$.

Moreover, serum testosterone levels were elevated 12 days after the initiation of metreleptin treatment and these remained elevated 1 year after the initiation of treatment. Additionally, serum testosterone levels have been reported to be elevated in leptin-overexpressing and leptin-treated mice $[13,14]$. This elevation was accompanied by a slight increase in skeletal muscle index one year after the initiation of metreleptin treatment. These findings indicate that long-term metreleptin treatment exerts secondary effects in addition to short-term effects, thus increasing skeletal muscle mass in GLPS patients. Generally, diet therapy is essential for diabetes, but there have been concerns on concomitant loss of skeletal muscle especially among patients with lipodystrophy. On the other hand, long-term testosterone therapy in men with hypogonadism increases muscle mass [15]. Therefore, elevation of serum testosterone levels by metreleptin treatment may benefit male patients by increasing skeletal muscle mass and subsequently improving metabolic profiles. Other than decreased skeletal muscle mass, our patient showed scoliosis as one form of musculoskeletal complication. Many GLPS patients show scoliosis and joint contractures [6] and one case report presented a GLPS patient with juvenile dermatomyositis [16]. There might be some involvement of the musculoskeletal system in the pathophysiology of GLPS and further investigation is thus warranted.

We confirmed the long-term increase in serum LH levels after leptin replacement in the present case. Previous reports showed that leptin could stimulate LH secretion not only in rodents but also in humans $[17,18]$ and leptin replacement rescued infertility of diabetic mice with an increase in serum LH and testosterone levels [19]. Moreover, low serum leptin levels are associated with hypothalamic amenorrhea and leptin replacement could rescue it with an increase in serum LH levels [20]. Therefore, the long-term elevation of serum testosterone levels in the present case might also be due to the effect of leptin replacement on the hypothalamic-pituitarygonadal (HPG) axis, which leads to an improvement in gonadal function. Unfortunately, we failed to assess whether hypogonadism in our patient was primary or secondary because he did not complain about gonadal function. We speculate that his hypogonadism was secondary because serum LH levels were low at the beginning of metreleptin replacement, and further investigation is necessary to reveal the effectiveness of leptin replacement in hypogonadism associated with lipodystrophy. The effect of leptin on the HPG axis is believed to be mostly via actions on the hypothalamus because the leptin receptor is abundantly expressed in hypothalamic neurons, but direct effects on the anterior pituitary gland or Leydig cells cannot be ruled out [21, 22]. Further investigation is necessary to reveal the detailed relationship between leptin and the HPG axis.

In conclusion, we herein reported the first Japanese case of GLPS with a de novo heterozygous LMNA p.T10I mutation. In addition, we evaluated short and long-term effectiveness of leptin replacement on generalized lipodystrophy by monitoring changes in serum testosterone levels along with the improvement in metabolic profiles. These findings indicate that testosterone might be involved in the metabolic effects of metreleptin and suggest that testosterone plays an important role in improving metabolic conditions of lipodystrophy patients.

\section{Disclosure}

None of the authors have any potential conflicts of interest associated with this case report.

\section{Ethical Approval}

We conducted whole exome sequence analysis with the Japanese nationwide medical research program for rare and intractable disease, IRUD (Initiative on Rare and Undiagnosed Diseases). It was done following informed consent of the patient, the approval of institutional review board of Tokyo Medical and Dental University research committee and the 1964 Helsinki declaration and its later amendments or comparable ethical standards.

\section{References}

1. Chiquette E, Oral EA, Garg A Araujo-Vilar D, Dhankhar P (2017) Estimating the prevalence of generalized and partial lipodystrophy: findings and challenges. Diabetes Metab Syndr Obes 13: 375-383.

2. Brown RJ, Arujo-Vilar D, Cheung PT, Dunger D, Garg A, et al. (2016) The diagnosis and management of lipodystrophy syndromes: a multi-society practice guideline. J Clin
Endocrinol Metab 101: 4500-4511.

3. Hennekam RC (2006) Hutchinson-Gilford progeria syndrome: review of the phenotype. Am J Med Genet A 140: 2603-2624.

4. Garg A, Subramanyam L, Agarwal AK, Simha V, Levine B et al. (2009) Atypical progeroid syndrome due to heterozygous missense LMNA mutations. J Clin Endocrinol 
Metab 94: 4971-4983.

5. Simha V, Agarwal AK, Oral EA, Fryns JP, Garg A (2003) Genetic and phenotypic heterogeneity in patients with mandibuloacral dysplasia-associated lipodystrophy. J Clin Endocrinol Metab 88: 2821-2824.

6. Hussain I, Patni N, Ueda M, Sorkina E, Valerio CM, et al. (2018) A novel generalized lipodystrophy-associated progeroid syndrome due to recurrent heterozygous LMNA p.T10I mutation. J Clin Endocrinol Metab 103: 10051014.

7. Oral EA, Simha V, Ruiz E, Andewelt A, Premkumar A, et al. (2002) Leptin-replacement therapy for lipodystrophy. N Engl J Med 346: 570-578.

8. Ebihara K, Kusakabe T, Hirata M, Masuzaki H, Miyanaga F, et al. (2007) Efficacy and safety of leptin-replacement therapy and possible mechanisms of leptin actions in patients with generalized lipodystrophy. J Clin Endocrinol Metab 92: 532-541.

9. Takeyari S, Takakuwa S, Miyata K, Yamamoto K, Nakayama H, et al. (2019) Metreleptin treatment for congenital generalized lipodystrophy type 4 (CGL4): a case report. Clin Pediatr Endocrinol 28: 1-7.

10. Godoy-Matos AF, Valerio CM, Moreira RO, Momesso DP, Bittencourt LK (2018) Pancreatic fat deposition is increased and related to beta-cell function in women with familial partial lipodystrophy. Diabetol Metab Syndr 10: 71.

11. Ogawa Y, Masuzaki H, Hosoda K, Aizawa-Abe M, Suga $\mathrm{J}$, et al. (1999) Increased glucose metabolism and insulin sensitivity in transgenic skinny mice overexpressing leptin. Diabetes 48: 1822-1829.

12. Fujikawa T, Chuang, JC, Sakata I, Ramadori G, Coppari R (2010) Leptin therapy improves insulin-deficient type 1 diabetes by CNS-dependent mechanisms in mice. Proc Natl Acad Sci U S A 107: 17391-17396.

13. Yura S, Ogawa Y, Sagawa N, Masuzaki H, Itoh H, et al.
(2000) Accelerated puberty and late-onset hypothalamic hypogonadism in female transgenic skinny mice overexpressing leptin. J Clin Invest 105: 749-755.

14. Mounzih K, Lu R, Chehab FF (1997) Leptin treatment rescues the sterility of genetically obese ob/ob males. Endocrinology 138: 1190-1193.

15. Traish AM, Haider A, Doros G, Saad F (2014) Long-term testosterone therapy in hypogonadal men ameliorates elements of the metabolic syndrome: an observational, longterm registry study. Int J Clin Pract 68: 314-329.

16. Sahinoz M, Khairi S, Cuttitta A, Brady GF, Rupani A, et al. (2018) Potential association of $L M N A$-associated generalized lipodystrophy with juvenile dermatomyositis. Clin Diabetes Endocrinol 4: 6.

17. Abel BS, Muniyappa R, Stratton P, Skarulis MC, Gorden $\mathrm{P}$, et al. (2016) Effects of recombinant human leptin (Metreleptin) on nocturnal luteinizing hormone secretion in lipodystrophy patients. Neuroendocrinology 103: 402407.

18. Zhang J, Gong M (2018) Review of the role of leptin in the regulation of male reproductive function. Andrologia 50: e12965.

19. Schoeller EL, Chi M, Drury A, Bertschinger A, Esakky P, et al. (2014) Leptin monotherapy rescues spermatogenesis in male Akita type 1 diabetic mice. Endocrinology 155: 2781-2786.

20. Welt CK, Chan JL, Bullen J, Murphy R, Smith P, et al. (2004) Recombinant human leptin in women with hypothalamic amenorrhea. N Engl J Med 351: 987-997.

21. Martin LJ, Roumaud P (2018) Modulation of the transcriptomic profile of the $\mathrm{R} 2 \mathrm{C}$ tumor Leydig cell line by the adipose tissue derived hormone leptin. Reprod Biol 18: 440-449.

22. Yu WH, Kimura M, Walczewska A, Karanth S, McCann SM (1997) Role of leptin in hypothalamic-pituitary function. Proc Natl Acad Sci U S A 94: 1023-1028. 